\title{
Interdependence of stress and interdiffusion during solid-state amorphization in $\mathrm{Ni}-\mathrm{Hf}$
}

\author{
W.S.L. Boyer \\ Department of Nuclear Engineering \& Radiological Sciences, University of Michigan, \\ Ann Arbor, Michigan 48109-2145 \\ M. Atzmon \\ Departments of Nuclear Engineering \& Radiological Sciences and Materials Science \& Engineering, \\ University of Michigan, Ann Arbor, Michigan 48109-2104
}

(Received 24 March 1999; accepted 17 November 1999)

\begin{abstract}
The evolution of stress in a Ni-Hf diffusion couple during solid-state amorphization reaction has been monitored by substrate curvature measurements and x-ray diffraction. The latter technique allowed an independent determination of the contribution of changes in stress-free lattice parameter to the stress in the crystalline layers. The results indicate that the amorphous phase forms under a large tensile stress, which relaxes as the reaction progresses. This stress in the amorphous phase is consistent with the volume change associated with the reaction. Stresses in the crystalline, elemental phases are considerably smaller and not affected by the reaction.

Low-temperature $\mathrm{Ni}$ ion irradiation increases the tensile stress in the diffusion couple. The large observed stress variations are not accompanied by variations in the effective interdiffusion coefficient.
\end{abstract}

\section{INTRODUCTION}

In the past decade, there has been much interest in solid-state routes of amorphous phase formation. One of these routes has been the solid-state amorphization reaction (SSAR). ${ }^{1}$ This growth of an amorphous layer by interdiffusion in bilayer diffusion couples has been observed in a large number of systems, ${ }^{1}$ typically characterized by a negative enthalpy of mixing and marked diffusional asymmetry. In such systems, temperature ranges exist which are both sufficiently high for the more-mobile species to undergo long-range diffusion but sufficiently low to kinetically suppress formation of equilibrium crystalline compounds by limiting rearrangement of the less-mobile species.

We have previously used diffusion couples consisting of a layer of as-deposited amorphous alloy in contact with one of the terminal phases. ${ }^{2}$ Alloy compositions that resulted in a vanishing reaction rate provided direct determination of the metastable common-tangent compositions. The interdiffusion coefficient, $\tilde{D}$, was shown to be quite sensitive to the compositon of the amorphous alloy. In the present work we study potential additional complications to the simple, constant $\tilde{D}$, description of SSAR kinetics.

Upon SSAR, an initially elemental bilayer diffusion couple evolves into a trilayer structure with the amorphous phase sandwiched between the two terminal phase layers. In the early stages, the amorphous layer thickness is generally observed to grow according to a $t^{1 / 2}$ rate law, in agreement with a diffusion-controlled mechanism., ${ }^{2,3}$ Some authors have reported negative deviations from the $t^{1 / 2}$ growth law in late reaction stages, occurring before the reactants were consumed. ${ }^{4-9}$ Both kinetic and thermodynamic explanations have been offered. One possible cause of a change in the rate law is an alteration in the macroscopic stress state. ${ }^{5,10-13}$ Stresses in asdeposited samples have been suggested to enhance the reaction rate in the early stages; as stresses are relieved during reaction, the reaction rate falls. ${ }^{5}$ Since there is generally a volume change upon amorphization, ${ }^{1,14}$ the reaction product is expected to be stressed, which can affect the atomic mobilities or the driving force.

In systems readily undergoing SSAR, in which there is substantial diffusional asymmetry, large microstresses (type III, or atomic-level stresses ${ }^{15}$ ), identified with a structurally unrelaxed state, are expected to evolve. ${ }^{16} \mathrm{In}$ contrast, the subject of the present study is macrostress. Macrostress here refers to type I stresses ${ }^{15}$ which are equilibrated within the sample and represent averages over volumes which include many grains but which may still be very small compared to the overall sample size. In diffusion couples consisting of amorphous $\mathrm{Ni}-\mathrm{Zr}$ alloy layers of differing compositions, observed dependence of the effective interdiffusion coefficient on the diffusion distance has been attributed to macrostresses, ${ }^{17}$ following a theoretical treatment by Stephenson. ${ }^{18}$ According 
to Stephenson, stress effects are significant for interdiffusion over small distance scales. In the present work, the amorphous layer thickness is initially zero and increases through the small length scale to large thicknesses. ${ }^{16}$

In the Co-Zr system, macroscopic apparent compressive stresses in crystalline $\mathrm{Zr}$ layers, based on $\theta / 2 \theta$ x-ray diffraction, combined with curvature measurements, suggest tensile stresses in the amorphous layers, which are associated with interdiffusion enhancement. ${ }^{11,12}$ However, in these studies, the lack of data on the stress-free lattice parameter and Co dissolution in the $\mathrm{Zr}$ layers complicate the interpretation. Aaen Andersen et al. observed behavior which suggests an effect of stress on the interdiffusion rate between as-deposited amorphous $\mathrm{Zr}-\mathrm{Co}$ and elemental Co. ${ }^{6}$ Their remarkable observations can be summarized as follows. After an initial period during which Co diffused into the amorphous alloy, the rate of change in the composition of the amorphous phase substantially decreased. The authors suggest that a steadystate composition was reached. It varied with reaction temperature more strongly than expected from the metastable free-energy diagram. Most importantly, samples which were irradiated after reaching a steady state resumed reaction upon further annealing. The authors suggest that, because of the significantly lower mobility of the $\mathrm{Zr}$, the indiffusion of Co led to microstress in the amorphous phase, resulting in a shift in its composition at metastable equilibrium with Co. Ion irradiation or higher temperatures, the authors argue, assisted relaxation of the amorphous phase, thus enabling the composition to more-closely approach metastable equilbrium.

These observations could be alternatively explained by a buildup of macroscopic stress-which was not monitored-during interdiffusion and its relaxation due to irradiation. Previous research addressing a stress effect on reaction rate is based on modeling rather than direct measurement. ${ }^{16,18}$ To improve our understanding of the interplay between macroscopic stress and diffusion in amorphous alloys, we have undertaken a study of diffusion and macroscopic stress during SSAR in Ni-Hf thin-film diffusion couples. In the present work, sampleaverage and crystalline layer stresses are directly measured. The Ni-Hf system ${ }^{2}$ was selected as a typical late transition metal-early transition metal couple for its chemical similarity to the widely-studied $\mathrm{Ni}-\mathrm{Zr}$ system and the large mass contrast which provides high resolution in Rutherford backscattering spectrometry (RBS) measurements. We report results of diffusion measurements by RBS and simultaneous stress measurements. Substrate curvature measurements yielded average film stress values, and $\mathrm{x}$-ray diffraction (XRD) in the Seemann-Bohlin geometry yielded phase-specific stress and stress-free lattice parameter values for the crystalline phases. The combination of the two stress measurements, plus the ability to independently determine changes in the stress-free lattice parameters and their contributions to stress, allowed us to separate the different contributions to stress in the crystalline layers. We have found there to be an offset between stresses measured by the two methods, in agreement with previous reports. ${ }^{19-23}$ This discrepancy does not affect our conclusions and is discussed only in this context. A full explanation of the discrepancy is beyond the scope of the present work. Low-temperature ion irradiation was used to modify the film stress without inducing additional mixing. We have found that significant stress changes occur in the amorphous phase as a result of either reaction or ion irradiation. However, these have not been accompanied by noticeable changes in the interdiffusion coefficient.

An essential feature of the present work is the consistent use throughout of control samples. Where the quantity of interest is stress in the amorphous layer of a reacting diffusion couple, stresses are also measured in single-layer elemental samples treated with the same time-temperature history. Where the quantities of interest are the stresses and reaction rate subsequent to ion irradiation, measurements are made immediately postirradiation. These are compared with an irradiated bare substrate and with an identical unirradiated diffusion couple reacted under the same conditions. Since the stresses relevant to the reaction are those present at the reaction temperature, values measured at room temperature are corrected to the annealing temperature. Uncertainties associated with this correction are shown to be small and so do not affect our conclusions. The essential quantities are not the stress magnitudes but the differences between control and experimental samples and the evolution of stress in the amorphous phase.

\section{EXPERIMENTAL METHODS AND ANALYSIS}

\section{A. Preparation}

Diffusion couples studied consisted of thin films of Ni and $\mathrm{Hf}$ on microscope slide or cover slip substrates 1.0 and $0.15 \mathrm{~mm}$ thick, respectively. Control samples consisted of elemental single layers on either substrate. Microscope slide substrates provided the rigid support needed for stable geometry in XRD measurements. Cover slip substrates are sufficiently thin to undergo easily measurable curvature changes due to typical film stresses.

Films were deposited by electron-beam evaporation in at a base pressure $<1 \times 10^{-8}$ torr; maximum pressures during deposition were in the low to mid $10^{-7}$ torr range. Film thicknesses were about 1500 to $3000 \AA$ and deposition rates about 1.5 to $5 \AA$ s. For samples with Ni nominally adjacent to the substrate ( $\mathrm{Ni}+\mathrm{Hf})$, a thin Hf layer was deposited directly on the substrate to improve adhesion. For samples with $\mathrm{Ni}$ as the nominal top layer 
$(\mathrm{Hf}+\mathrm{Ni}$ ), a thin Hf cap was deposited on the surface to protect against oxidation. ${ }^{4}$ Resulting diffusion couples consisted of substrate $+25 \AA \mathrm{Hf}+2000 \AA \mathrm{Ni}+3000 \AA$ $\mathrm{Hf}(\mathrm{Ni}+\mathrm{Hf})$ or substrate $+3000 \AA \mathrm{Hf}+1500 \AA \mathrm{Ni}+$ 100 A $\mathrm{Hf}(\mathrm{Hf}+\mathrm{Ni})$, approximately.

Samples were reacted in flowing Ti-gettered $\mathrm{Ar}$ at $340{ }^{\circ} \mathrm{C}$ for up to $540 \mathrm{~min}$ with interruptions for characterization. To minimize oxidation, samples were buried under $\mathrm{Zr}$ powder and the chamber was purged for at least $24 \mathrm{~h}$ before annealing. Temperatures were controlled to within about $\pm 0.2^{\circ}$ and times to about $\pm 2 \mathrm{~min}$.

In order to observe the effect of irradiation on stress and interdiffusion, two Ni-Hf diffusion couples on cover slip substrates were deposited and annealed simultaneously up to $156 \mathrm{~min}$. One of the two was then irradiated with $2.9 \times 10^{14} 3.0 \mathrm{MeV} \mathrm{Ni}^{+2} \mathrm{~cm}^{-2}$, leading to about 0.5 displacements per atom, as calculated by TRIM $;{ }^{24}$ the other sample provided a control. Subsequent anneals of the two samples were also performed simultaneously. Chemical effects in the film are negligible since the ion dose used is equivalent to $0.3 \mathrm{Ni}$ atomic monolayers, and $>95 \%$ the ions fully penetrate the metal film, coming to rest in the substrate (from simulation). To avoid straining the sample, it was loosely mounted to a heat sink and irradiated for $20 \mathrm{~h}$ at a power density of only $4 \mathrm{~mW} / \mathrm{cm}^{2}$ while continuously cooled with liquid $\mathrm{N}_{2}$. The irradiation was determined to bring the sample temperature to $\sim 80 \mathrm{~K}$.

Since nearly all of the incident ions fully penetrate the metal layers, and irradiation has been observed to cause expansion $^{25}$ or contraction ${ }^{26}$ in glasses, any curvature changes upon irradiation could be due to film or substrate changes. Therefore a control was required, which consisted of $80 \AA$ Hf on a cover slip substrate. The thin Hf layer provided electrical conductivity and mimicked the film-substrate interface portion of the sample. This control was irradiated to the same dose using the same species, power density, and cooling methods as the sample. The energy used, $1.5 \mathrm{MeV}$, corresponded to the average energy of ions exiting the back of the metal film layers (from TRIM). ${ }^{24}$

\section{B. Characterization}

Rutherford backscattering spectrometry, RBS, using $2-\mathrm{MeV} \mathrm{He}^{2+}$ at a scattering angle of $165^{\circ}$ or $170^{\circ}$, was performed after each treatment. Spectra from $\mathrm{Au}$ and $\mathrm{Si}$ were acquired along with sample data for detector and electronics calibration. RUMP ${ }^{27}$ was used to obtain nonlinear least-squares fits (LSF) between sample descriptions and the most relevant portions of each spectrum, yielding atomic composition as a function of depth and thus individual layer thicknesses. To correct for small discrepancies in the measured total metal content, layer thicknesses were normalized to constant total metal con- tent. The oxygen concentration was observed by acquiring RBS data with sufficient statistics and carefully fitting the spectrum. In all cases, no measurable oxygen content was observed within at least $1500 \AA$ of the interface.

Both diffusion couples and single-layer elemental controls were characterized by XRD in order to determine the phases present and measure the stress evolution in the crystalline phases. A Seemann-Bohlin ${ }^{28}$ thin-film diffractometer (TFD) was used. In this geometry, the angle of incidence, $\gamma$, is fixed, usually at $\gamma=10^{\circ}$, and the detector scans $2 \theta$. For the present film thickness and $\gamma$, the $\mathrm{x}$-ray signal uniformly samples the entire film depth. Since the direction of the scattering vector varies with $2 \theta$, each scan provides the lattice parameter, $d$, for many crystallite orientations. XRD was performed primarily on microscope slide substrates; consistency checks were performed on some of the cover slip samples using a custom-made sample holder designed to avoid straining them.

To measure biaxial stress, careful calibration of the TFD was required. A correction function for angular aberrations was obtained by fitting the diffraction angles of an $\mathrm{Au}$ or $\mathrm{LaB}_{6}$ standard $\left(\mathrm{LaB}_{6}\right.$ powder embedded in epoxy with lapped and polished surface supplied by Gem Dugout, State College, PA) to the functional form expected for instrumental angular deviations ${ }^{29}$ plus an empirical linear term. Diffraction peaks were fit to an asymmetric Pearson VII function $^{30}$ plus background. In the sample reference frame, a biaxial stress, $\sigma_{x}$, is assumed $\left(\sigma_{x}=\sigma_{x x}=\sigma_{y y}\right.$; all other $\left.\sigma_{i j}=0\right)$ since deposited atoms were nearly normally incident on an amorphous substrate. In this case the strain component is $\epsilon_{\psi}=\left(d-d_{0}\right) / d_{0}$, where $\psi=\theta-\gamma$ is the angle between the diffraction vector and the sample normal, $d_{0}$, the reference pure-material stress-free spacing for the given reflection and $d$, the XRD-measured lattice spacing at $\psi$. Full tensor analysis was used to derive $e^{31-33} \epsilon_{\psi}=$ $f_{\epsilon}\left(\sigma_{x}, h k l\right)+\delta_{\mathrm{H}}$, assuming uniform stress and accounting for anisotropic linear elastic constants. Here $f_{\epsilon}$ is a discrete function of the Miller indices $(h k l)$ and the compliance tensor, and $\delta_{\mathrm{H}}$ is the isotropic ("hydrostatic") relative deviation from the pure-material stress-free lattice spacings. Values of $\sigma_{x}, \delta_{\mathrm{H}}$, and uncertainties were obtained by nonlinear LSF to statistically weighted $\epsilon_{\psi}$; most $\sigma_{x}$ and $\delta_{\mathrm{H}}$ values were averaged over repeated scans or samples. An example of the fit to $\epsilon_{\psi}$ is shown in Fig. 1, in which the strain is plotted versus $\sin ^{2}(\psi)$. For an isotropic material, this plot would give a straight line ${ }^{15}$ with slope proportional to the stress and with stress-free lattice parameter observed at $\psi_{0}=2 v /(1+v)$; i.e., $\epsilon_{\psi_{0}}=\delta_{\mathrm{H}}$, where $v$ is Poisson's ratio. ${ }^{34} \mathrm{We}$ note here that the ability to determine the stress-free lattice parameter allows us to determine the contribution of solute incorporation to the observed stress. 


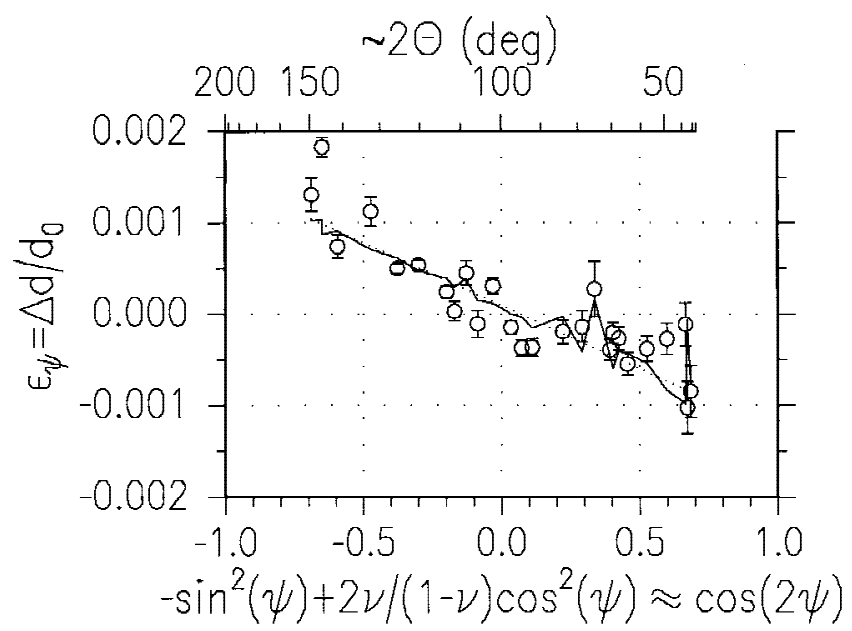

FIG. 1. Strain versus $\sin ^{2}(\psi)$ with $\gamma=10^{\circ}$ for the Hf phase of a diffusion couple. In this geometry, $\psi=\theta-\gamma$. The solid line is the nonlinear fit to the discrete function $f_{\epsilon}\left(\sigma_{x}, h k l\right)+\delta_{\mathrm{H}}$, weighted according to the error of each data point.

Estimates of grain size and root-mean-square strain, $\epsilon_{\text {rms }}$, were also obtained from the XRD data. The net integral breadth is calculated from the observed and instrumental integral breadths, $w_{\text {obs }}$ and $w_{\text {inst }}$, respectively, by $\beta=\left\{\left(w_{\text {obs }}\right)^{2}-\left(w_{\text {inst }}\right)^{2}\right\}^{1 / 2}$. A weighted LSF to a plot of $2 \sin (\theta) / \lambda$ versus $2 \beta \sin (\theta) / \lambda$ yields grain size $=1 /$ intercept and $\epsilon_{\mathrm{rms}}=$ slope/2.5. ${ }^{35}$

Stresses were also determined from curvature changes of cover slip samples. All cover slip substrates were initially selected by optical interferometry for small and uniform deviations from flatness. Curvatures were measured on a Dektak profilometer in two orthogonal directions. For validation, we compared the Dektak measurement with optical interferometry ${ }^{36}$ and laser $^{37}$ profilometry and found them to agree to within about $\pm 0.005 \mathrm{~m}^{-1}$. The $15 \mathrm{mg}$ mass-equivalent force applied by the scanning stylus is calculated to cause negligible deflection. Samples were held in a custom-made support to prevent sliding, rocking, or straining. Measured profiles were fitted to radii of curvature, $R$, over the central $14 \mathrm{~mm}$, using instrument software. Typical deflections over the scan were about 1.5 to $20 \mu \mathrm{m}$ from flat, corresponding respectively to curvatures, $K=1 / R$, of about 0.06 to $0.8 \mathrm{~m}^{-1}$. The equation used for the biaxial film stress, $\sigma_{x}$, corrected for plate geometry and modified to use separate measurements in the $x$ and $y$ directions, is ${ }^{36}$

$$
\sigma_{x}=\frac{E_{\mathrm{s}} t_{\mathrm{s}}^{2}}{6\left(1-v_{\mathrm{s}}\right) t_{\mathrm{f}}}\left(\Delta K_{x}+\Delta K_{y}\right)
$$

Subscripts indicate substrate (s) or film (f) quantities, $E$ is Young's modulus, and $t$ is the film thickness. $\Delta K_{\alpha}=$ $1 / R_{\alpha}-1 / R_{\alpha 0}$, where $R_{\alpha}$ is the radius of curvature of the sample in the $\alpha$ direction ( $x$ or $y$ ) and $R_{\alpha 0}$ that of the substrate before deposition. For the film and cover slip thicknesses used and a maximum deflection of about $40 \mu \mathrm{m} \approx 0.25 t_{\mathrm{s}}$, the assumptions made in deriving Eq. (1) are well-satisfied. ${ }^{38}$

\section{RESULTS}

\section{A. Stresses}

The biaxial stress in the crystalline layers, determined by XRD at room temperature, has been corrected for thermal stress to determine the stress at the annealing temperature, $T_{\text {ann }}$ (assuming no relaxation or yield during cooldown, see Sec. IV. B. 1 for further discussion). These corrected stresses and relative changes in purematerial stress-free lattice spacing, $\delta_{\mathrm{H}}$, are displayed as a function of $t^{1 / 2}$ in Figs. 2(a) and 2(b) for $\mathrm{Ni}$ and $\mathrm{Hf}$, respectively. Following convention, positive stresses and strains are tensile. In as-deposited Ni layers, $\delta_{\mathrm{H}}$ is about $(+0.19 \pm 0.02) \%$ and $(+0.23 \pm 0.03) \%$ in single-layer elemental and diffusion couple samples, respectively. In both, $\delta_{\mathrm{H}}$ increases by about $0.05 \%$ from the initial value in the first $30 \mathrm{~min}$ of annealing, followed by little subsequent change. The biaxial stress at room temperature, $\sigma_{\text {obs }}$, in the Ni phase is initially $\sigma_{\text {obs }}=-0.08 \pm 0.06$ and $0.28 \pm 0.06 \mathrm{GPa}$ in single-layer elemental and diffusion couple samples, respectively, increases by about $0.30 \mathrm{GPa}$ in the first $30 \mathrm{~min}$ of annealing in both sample types and then approximately saturates.

In as-deposited $\mathrm{Hf}$ layers, $\delta_{\mathrm{H}}$ is about $(+0.08 \pm 0.01) \%$ and $(+0.04 \pm 0.01) \%$ in the single-layer elemental and diffusion couple samples, respectively. In both, $\delta_{\mathrm{H}}$ increases by about $0.08 \%$ in the first $30 \mathrm{~min}$ of annealing, followed by little subsequent change. The Hf phase is initially under a small tensile stress which decreases and becomes compressive upon annealing. Initially $\delta_{\text {obs }}$ in the Hf phase in the single-layer elemental and diffusion couples samples is the same, $+0.12 \pm 0.09$ and $0.15 \pm$ $0.02 \mathrm{GPa}$, respectively. In both samples types, $\sigma_{\text {obs }}$ decreases by about $0.21 \mathrm{GPa}$ and reaches about $-0.08 \pm$ $0.03 \mathrm{GPa}$ in the first $30 \mathrm{~min}$ of annealing and then saturates. In summary, within error, the same changes in $\sigma_{\mathrm{obs}}$ and $\delta_{\mathrm{H}}$ occur in the two sample types of $\mathrm{Ni}$ and $\mathrm{Hf}$ over the annealing times studied.

The evolution of stresses with anneal time at $340{ }^{\circ} \mathrm{C}$ was also measured in one diffusion couple consisting of opposite layer order, $\mathrm{Hf}+\mathrm{Ni}$ (not shown). Within error bars, the initial values of $\sigma_{x}$ and $\delta_{\mathrm{H}}$ and their evolution were the same in $\mathrm{Ni}$ layers of both the $\mathrm{Hf}+\mathrm{Ni}$ and $\mathrm{Ni}+$ $\mathrm{Hf}$ samples. In the $\mathrm{Hf}$ layers, such agreement is also observed for the $\delta_{\mathrm{H}}$ values; however the initial $\sigma_{x}$ values are greater in the $\mathrm{Ni}+\mathrm{Hf}$ sample and decrease to a lower value after annealing 30 min compared to the $\mathrm{Hf}+\mathrm{Ni}$ sample. In view of the qualitative agreement and larger error bars on the $\mathrm{Hf}+\mathrm{Ni}$ sample $\sigma_{x}$ data, the observed differences are not likely significant. At longer times, no significant differences are observed. When comparing 


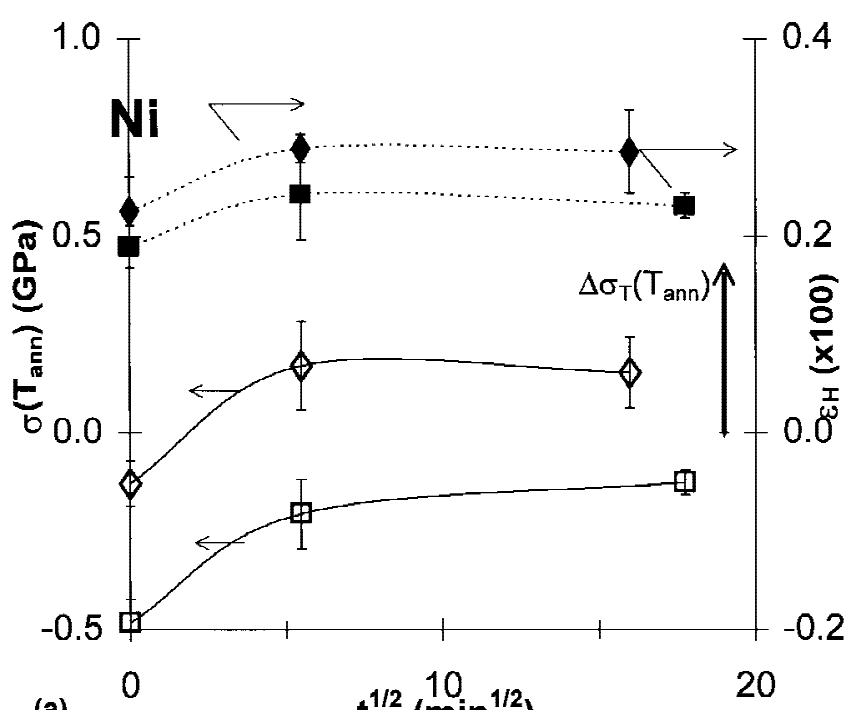

(a)

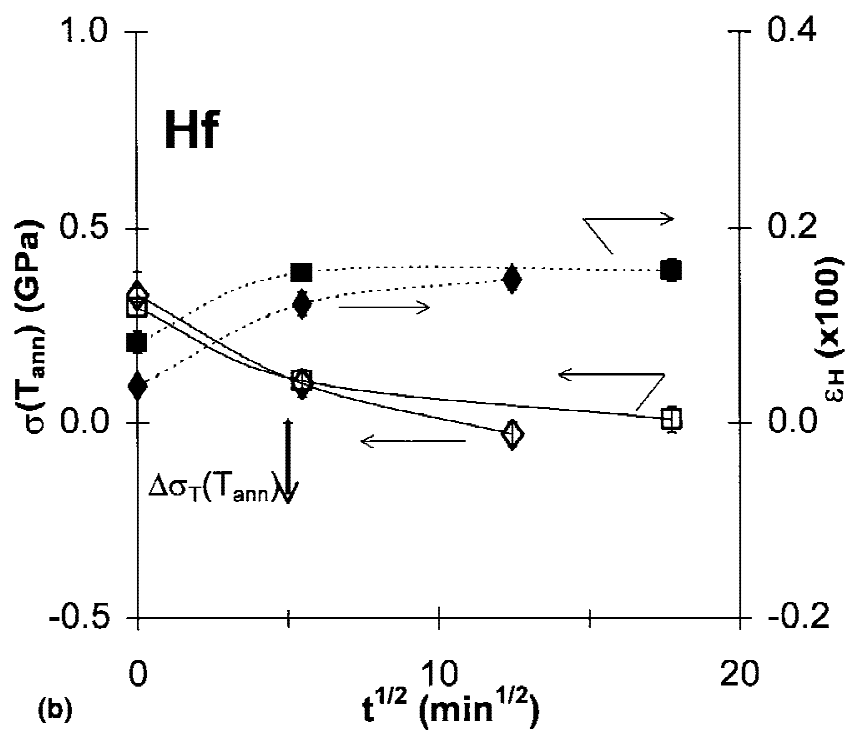

FIG. 2. Biaxial stress at $T_{\text {ann }}\left(340{ }^{\circ} \mathrm{C}\right), \sigma\left(T_{\text {ann }}\right)$, calculated from XRD measurements and differential thermal expansion (open symbols) and relative change in pure-material stress-free lattice spacing, $\delta_{\mathrm{H}}$ (solid symbols), in crystalline layers of diffusion couple $(\diamond, \diamond)$ and singlelayer elemental samples ( $\square, \mathbf{\square})$ : (a) Ni phase, (b) Hf phase. $\Delta \sigma_{\mathrm{T}}\left(T_{\text {ann }}\right)$ indicates the thermal stress upon cooling from $T_{\text {ann }}$ to room temperature (see discussion). If relaxation or yield occur during cooldown, the stress curves may be shifted by an offset up to $\Delta \sigma_{\mathrm{T}}\left(T_{\text {ann }}\right)$ in the direction indicated by the arrow.

some additional, nominally identical samples, made in separate depositions, significant scatter in as-deposited $\sigma_{x}$ and $\delta_{\mathrm{H}}$ values is observed. However, in most-cases, similar changes are observed in the first $30 \mathrm{~min}$. In all cases, the same changes, within error, are observed from $30 \mathrm{~min}$ on.

Biaxial stress results obtained from curvature for a pair of 5200- $\AA$-thick Ni-Hf diffusion couples are shown in Fig. 3; stresses shown are corrected to $T_{\text {ann }}$ (assuming no relaxation or yield during cooldown, see Secs. IV. B. 1

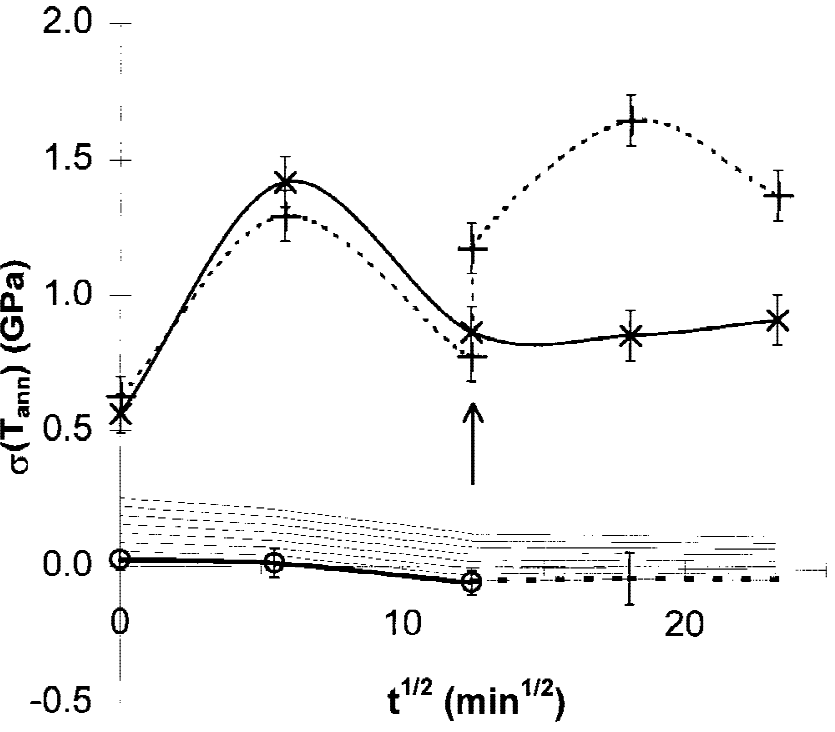

FIG. 3. Average stresses in Ni-Hf bilayer diffusion couples on cover slip substrates as a function of reaction time obtained from curvature and corrected to $T_{\text {ann }}=340{ }^{\circ} \mathrm{C}$ : annealed only $(\mathrm{x})$; annealed and irradiated at time indicated by arrow (+). The contribution of the crystalline layers, calculated from data of Figs. 2 and 4, plus that of thermal stress in the amorphous layer is also displayed $(\bigcirc$, dashed portion by extrapolation); the band consisting of closely spaced parallel lines indicates the possible range if thermal stresses in the crystalline layers partially relax or yield during cooldown; see discussion.

and IV. C. 2). These two samples were annealed together at $340{ }^{\circ} \mathrm{C}$ for $156 \mathrm{~min}$, one was irradiated, and they were then further annealed together. In the sample that was annealed only (see below for irradiation), the measured $\sigma_{x}$ was initially about $0.77 \mathrm{GPa}$, increased to about $1.5 \mathrm{GPa}$ after $30 \mathrm{~min}$ at $340{ }^{\circ} \mathrm{C}$, and then decreased and saturated at about $1.0 \mathrm{GPa}$.

For as-deposited single-layer elemental samples there is variation of up to $1 \mathrm{GPa}$ between stress measurements by XRD and curvature. One single-layer elemental sample (Ni) was annealed, and stresses were measured by both methods: within the uncertainty, the two techniques indicate the same stress changes. The expected and observed agreement between the two measurement methods are discussed below (Sec. IV. A).

\section{B. Other XRD results}

Grain size and root mean square strain $\left(\epsilon_{\mathrm{rms}}\right)$ values have been determined as described above. The initial grain size is $\sim 500 \AA$ in $\mathrm{Ni}$ and $\sim 300 \AA$ in Hf. When annealed for $5 \mathrm{~h}$ at $340{ }^{\circ} \mathrm{C}$, the grain size in $\mathrm{Ni}$ increases to $\sim 600 \AA$, but that of Hf remains unchanged, within uncertainty. Initially, $\epsilon_{\mathrm{rms}}$ is $\sim 0.1 \%$ and $\sim 0.2 \%$ in $\mathrm{Ni}$ and Hf, respectively. Upon annealing, $\epsilon_{\mathrm{rms}}$ in $\mathrm{Ni}$ decreases to about half of its initial value; in Hf the value remains constant, within uncertainty. Since the XRD data were 
not originally collected for grain size and $\epsilon_{\mathrm{rms}}$ determination, the uncertainties in the results are large and only the relative magnitudes and qualitative changes are likely significant.

$\mathrm{X}$-ray scans taken in the $\theta / 2 \theta$ geometry give a general indication of texture. Ni shows all of the expected peaks with roughly the expected intensities for randomly polycrystalline material, indicating, at most, weak texture. In Hf films, on the other hand, only a few weak peaks aside from (002) and its multiples are observed, indicating a significant (001) preferred orientation.

\section{Composition profiles}

In Fig. 4 the amorphous layer thickness, $d_{\mathrm{am}}$, is plotted versus $t^{1 / 2}$ for the same samples as Fig. 3 and the $\mathrm{Hf}+\mathrm{Ni}$ sample; the slope squared is directly proportional to the effective interdiffusion coefficient, $\tilde{D}^{39}$ On the basis of our previously-determined common tangent compositions $^{2}$ and the initial slope, $\tilde{D}=1.6 \times 10^{-19} \mathrm{~m}^{2} / \mathrm{s}$.

We observe that neither the changes in average stress (Fig. 3) nor stress changes in individual layers (Fig. 2) are accompanied by any noticeable change in the interdiffusion coefficient. A comparison of the $\mathrm{Hf}+\mathrm{Ni}$ curve with that of $\mathrm{Ni}+\mathrm{Hf}$ in Fig. 4 is suggestive that the reaction rate may be influenced by layer order. However, while the $\mathrm{Hf}+\mathrm{Ni}$ sample has a greater initial reaction rate, at later times the two curves become parallel within uncertainty. Finally, note that $\tilde{D}$ decreases slightly at late times.

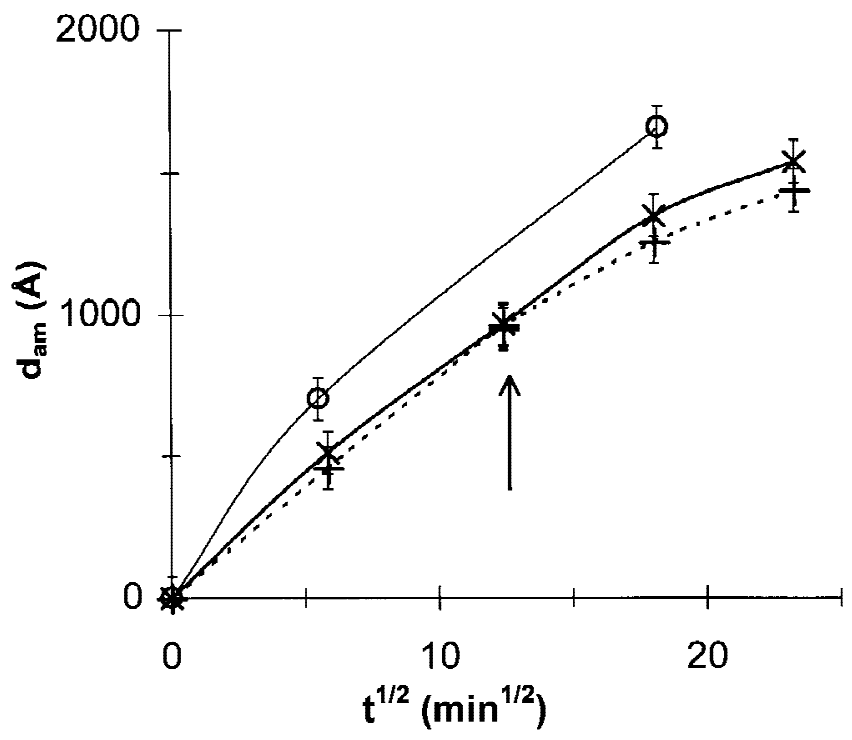

FIG. 4. Amorphous layer thickness, $d_{\mathrm{am}}$, obtained from RBS, as a function of reaction time at $340{ }^{\circ} \mathrm{C}$. $\mathrm{Ni}+\mathrm{Hf}$ layer order, same samples as Fig. 3: annealed only ( $\mathrm{x}$ ); annealed and irradiated (arrow) with $d_{\mathrm{am}}$ measured before and after irradiation $(+)$; also $\mathrm{Hf}+\mathrm{Ni}$ annealed only $(\bigcirc)$.

\section{Irradiation}

As described above, one of the partially reacted diffusion couples was irradiated at low temperature using $\mathrm{Ni}^{2+}$ ions of sufficient energy to fully penetrate the films. Figure 3 displays the average sample biaxial stress as a function of time with the time of irradiation indicated. Curvature and RBS measurements were made subsequent to each anneal and immediately postirradiation. The tensile stress increases significantly upon irradiation. The control substrate curvature change indicates that only about $20 \%$ of the apparent stress change is due to a direct irradiation effect on the substrate. Annealing the irradiated control substrate also shows that little of the apparent stress change upon irradiation or postirradiation annealing is due to a direct effect on the substrate. The net tensile stress increase due to irradiation effects on the metal layers is about $0.32 \mathrm{GPa}$; the increase due to subsequent annealing is $0.48 \mathrm{GPa}$. On the basis of preliminary XRD results subsequent to postirradiation annealing, there is no difference in Ni layer stress between irradiated and unirradiated samples; $\delta_{\mathrm{H}}$ is slightly less in the irradiated sample. In the Hf layer, $\sigma_{x}$ increases by $0.43 \mathrm{GPa}$ following irradiation plus anneal, from compressive to tensile values; $\delta_{\mathrm{H}}$ increases only very slightly.

The impact of irradiation on reaction extent can be seen in Fig. 4, in which the irradiation time is indicated. As remeasured by RBS immediately after irradiation, no detectable change in the composition profile resulted from irradiation (data points overlap in the figure). Subsequent to the irradiation, the irradiated and unirradiated samples were annealed further. To good precision, no difference in postirradiation reaction rate is detected; the two Ni + Hf curves of Fig. 4 are within error bars of one another. In summary, we observe that the stress changes due to interdiffusion, irradiation, or additional postirradiation reaction are not accompanied by a significant change in reaction rate.

\section{DISCUSSION}

For both Ni and Hf, in single-layer elemental and in diffusion couple samples with either layer order, we find that while differences exist in the as-deposited values, the changes in biaxial stress and stress-free lattice parameter with time are the same within error. We suggest that differences between nominally identical, as-deposited samples are due to insufficient control of some deposition variable(s). The similar evolution of nominally identical samples gives us confidence in the stress and strain measurements. A source of concern is the observed disagreement between XRD and curvature measurements; such disagreement has been previously reported by other authors and is discussed in Sec. IV. A. It is concluded that while the two methods disagree on stress in asdeposited samples, they agree on stress evolution. There- 
fore, their combined use for the crystalline phases allows us to determine the stress evolution in the amorphous phase. In section IV. B, thermal and solute contributions to stresses are analyzed. Observed stress changes in the crystalline and amorphous phases are discussed in Secs. IV. C. 1 and IV. C. 2, respectively. Section IV. D deals with the irradiation and its effects on stress and diffusion. Finally, the diffusion kinetics are discussed in Sec. IV. E.

\section{A. Comparison of XRD and curvature stress measurements}

While the two stress measurement methods used in the present work agree on stress evolution, they disagree in magnitude; this section discusses the discrepancy. The average stress, $\bar{\sigma}$, in a film consisting of more than one layer is the thickness-weighted average of individual layer stresses, $\sigma_{i}$, plus contributions from each interface,

$$
\bar{\sigma}=\left[\sum_{j}^{M} \gamma_{j}+\sum_{i}^{N} \sigma_{i} x_{i}\right] / \sum_{i}^{N} x_{i}
$$

where $\gamma_{j}$ is the interfacial stress, $M$ is the number of interfaces, and $N$ the number of layers, each of thickness $x_{i}$. The interfacial contribution is negligible for the small number of interfaces in our samples. ${ }^{40}$ No direct measurement of stresses in the amorphous layer is possible in the present samples. A combination of XRD and substrate curvature measurements has been used by other authors ${ }^{12,40-42}$ to determine the contributions to the stress that are not directly observable. However, great care should be taken in basing conclusions on differences in stress values measured by the two techniques. On first consideration, one expects the stress in the amorphous phase to be the difference between $\sigma_{x}$ from curvature measurement and the contributions of the crystalline phases, where the latter are calculated using Eq. (2), XRD mearusements of $\sigma_{i}$ (Fig. 2), and RBS measurements of $x_{i}$ (Fig. 4). However, in the as-deposited diffusion couples, where the amorphous layer thickness is negligible, the average stress calculated by Eq. (2) does not agree with that of the curvature measurements. The discrepancy is greater than reasonably explained by interfacial stress. As stated above, we observe similar discrepancies in single-layer elemental samples. Consequently, it is not possible to quantitatively calculate the stress in the amorphous phase. However, in light of our comparison of measurements on the single-layer elemental controls by both methods, we conclude below that the measured curvature change is dominated by the contribution of the stress in the amorphous layer.

We now discuss possible sources of disagreement between the two stress measurement methods. The assumption that the same biaxial stress tensor describes all crystallites in the film (Reuss average) is one source of error, since it fails to satisfy material continuity. ${ }^{43}$ Taking single-crystal anisotropy into account does not significantly improve the fit in Fig. 1. The discrepancy between the observed strain anisotropy and that calculated by $f_{\epsilon}$ possibly results from the particular texture of the sample (random texture is assumed in the derivation). Note that approximations other than that made by Reuss ${ }^{43}$ would yield even less calculated strain anisotropy. The additional complexity of including texture in the full-tensor derivation of $f_{\epsilon}$ is not warranted in the present work.

In a fine-grained polycrystalline sample, generally, a small fraction of all grains will be suitably oriented for diffraction. If the grains are randomly oriented, the signal is representative of the overall average. However, in textured samples, the fraction of grains diffracting may be much smaller and nonrepresentative of the overall average. While some authors have reported quantitative agreement between the two techniques,${ }^{44}$ careful comparison has revealed frequent disagreement. ${ }^{19-22}$ It should be noted that the disagreement is not likely to be caused by selective sampling of the crystallite population because Malhotra et al. have found significant disagreement when sampling the majority of the population in a textured film. ${ }^{20,21}$ The fact that each data point in Fig. 1 corresponds to a different part of the crystallite population contributes to the scatter but is very unlikely to introduce a systematic error in the slope. Disagreement was also observed in nearly texture-free films ${ }^{20,21}$ including the Ni films in the present work.

While there is disagreement between our two methods, the difference remains about constant with annealing time. In order to address whether the extent of agreement would be expected to change with annealing, one must consider whether the crystallite orientation distribution can be significantly altered. Recrystallization could substantially alter the orientation distribution, but the moderate change in grain size we observe indicates insignificant recrystallization. The agreement could also change with time if the grain orientation distribution evolves due to abnormal or secondary grain growth, common in thin films. ${ }^{45}$ While not satisfactorily explained by the authors, anneals causing recrystallization and/or grain growth have been observed to cause an increasing discrepancy between XRD and curvature stress measurements. ${ }^{23}$ In the present work, the extent of grain growth is small and is not expected to significantly alter the agreement between the two measurement methods. Plastic deformation could also affect the extent of agreement ${ }^{15,46}$ by changing the grain orientations. While sample processing performed by Venkatraman ${ }^{44}$ does induce plastic deformation, the extent is small, $\epsilon<1 \%$, and is not expected or observed to change the agreement between the two measurement methods. In the present work, total thermal strains are also small, $0.2 \%$, and 
strains in the crystalline layers due to stress in the amorphous layer are negligible. Therefore, plastic deformation is not expected to significantly alter grain shapes or orientations.

In conclusion, stress measurements by curvature and diffraction techniques are expected to quantitatively agree for random crystallite orientations. However, disagreement due to nonrandom grain orientation distributions may be much more common than is generally appreciated. ${ }^{19-23,43,47}$ In the present work and reported cases of disagreement, ${ }^{19,20,23}$ more tensile stresses are often observed by the curvature technique. Both methods are expected to indicate the same stress evolution under themal treatments like that of the present work and Ref. 44 as long as neither recrystallization, nor grain growth, nor plastic deformation is substantial.

\section{B. Stress due to $\delta_{\mathrm{H}} \neq 0$}

Calculated contributions to the biaxial stress resulting from changes in the lattice parameter will be addressed in this section. In a thin film attached to a thicker substrate, biaxial stresses will generally result from any differential volume change between film and substrate. The biaxial stress, $\sigma_{x}$, arising from a relative change in the stress-free lattice parameter, $\delta_{\mathrm{H}}$, is ${ }^{48}$

$$
\sigma_{x}\left(\delta_{\mathrm{H}}\right)=-\frac{E}{1-v} \delta_{\mathrm{H}}
$$

Several physical processes contribute to $\delta_{\mathrm{H}}$. They include differential thermal expansion, solute incorporation, and various structural changes in the elemental films. Contributions to the stresses we observe are discussed below. Calculations of thermal, impurity, and structural change effects on stresses in the crystalline phases are presented in Secs. IV. B. 1, IV. B. 2, and IV. C. 1, respectively. Application to the present work and interpretation is discussed in Sec. IV. C.

\section{Thermal stress}

The stress at the annealing temperature is given by $\sigma\left(T_{\text {ann }}\right)=\sigma_{\text {obs }}-\Delta \sigma_{\mathrm{T}}\left(T_{\text {ann }}\right)$, where $\Delta \sigma_{\mathrm{T}}(T)=E\left(\alpha_{\mathrm{f}}-\alpha_{\mathrm{s}}\right)$ $\left(T-T_{\text {obs }}\right) /(1-v)$ is the thermal stress. ${ }^{48}$ Little stress relaxation or yield are assumed to occur at either room temperature, $T_{\mathrm{obs}}$, or in the few minutes required for the heating or cooling. In Fig. 2, $\sigma\left(T_{\text {ann }}\right)$ is displayed; $\Delta \sigma_{\mathrm{T}}\left(T_{\mathrm{ann}}\right)$ is indicated by an arrow. If stress relaxation or yield occurs at room temperature or during cooldown, the $\sigma\left(T_{\text {ann }}\right)$ values prior to cooldown will be shifted by about the same amount at each point, up to a maximum of $\Delta \sigma_{\mathrm{T}}\left(T_{\mathrm{ann}}\right)$, in the direction indicated by the arrow. We have analyzed all possible combinations of heating, cooling, and initial stress state to determine when relaxation or yield due to thermal stress are possible. We conclude that this is only the case during cooldown, since, upon heating, thermal stresses are either small or cause the stress magnitude to decrease. The following literature values were used: Linear coefficients of thermal expansion for $\mathrm{Ni},{ }^{49} \mathrm{Hf},{ }^{49}$ microscope slides, ${ }^{50}$ and cover slip ${ }^{50}$ are $\alpha_{\mathrm{Ni}}=13.3 \times 10^{-6} \mathrm{~K}^{-1}, \alpha_{\mathrm{Hf}}=6.0 \times 10^{-6} \mathrm{~K}^{-1}$, $\alpha_{\mathrm{MS}}=8.9 \times 10^{-6} \mathrm{~K}^{-1}$, and $\alpha_{\mathrm{CS}}=7.38 \times 10^{-6} \mathrm{~K}^{-1}$, respectively; also $E_{\mathrm{Ni}}=200 \mathrm{GPa}, \nu_{\mathrm{Ni}}=0.31, E_{\mathrm{Hf}}=$ $141 \mathrm{GPa}$, and $\nu_{\mathrm{Hf}}=0.26 .{ }^{49}$ See below for the thermal expansion of the amorphous phase.

\section{Stress resulting from solute incorporation}

Dissolution of gaseous or metal impurities will cause a change in the stress-free lattice parameter, resulting in $\delta_{\mathrm{H}} \neq 0$. If impurities causing $\delta_{\mathrm{H}}>0$ are incorporated during deposition, before the metal atoms become adherent to the remainder of the film + substrate, no macroscopic stress will result. Impurities entering the film after it forms will cause biaxial stress changes $\sigma_{x}\left(\delta_{H}\right)$ given by Eq. (3). Note that our ability to independently measure the stress-free lattice parameter, and thus determine $\delta_{\mathrm{H}}$, enables us to determine this stress contribution.

\section{Discussion of observed stresses}

This section addresses mechanisms giving rise to observed stress changes in the present work. In IV. C. 1, we establish that the contribution of the crystalline layers to the observed average stress is small. In IV. C. 2, we discuss the stress changes in the amorphous phase.

\section{1. $\sigma_{x}$ and $\delta_{H}$ in the crystalline phases}

In the present work, generally $\delta_{\mathrm{H}}>0$; this is qualitatively consistent with interstital solute incorporation, such as $\mathrm{Ni}$ in $\mathrm{Hf}$ or gaseous impurities in either metal or with large-atom substitutional impurities, such as $\mathrm{Hf}$ in Ni. The single-layer elemental samples exhibit about the same $\delta_{\mathrm{H}}$ values and evolution as the diffusion couples in both as-deposited and annealed cases. This control enables us to rule out dissolution of $\mathrm{Ni}$ in $\mathrm{Hf}, \mathrm{Hf}$ in $\mathrm{Ni}$, or any other effects of the SSAR as sources of $\delta_{\mathrm{H}}$ in the diffusion couples. Absorption of 2 at.\% oxygen during the annealing of $\mathrm{Hf}$ is quantitatively consistent with the observed $\delta_{\mathrm{H}}{ }^{51}$ and is plausible, given the reactivity of Hf. That $\delta_{\mathrm{H}} \neq 0$ in as-deposited Ni suggests the presence of impurities in the as-deposited film. While the authors are not aware of quantitative studies of Ni lattice parameter as a function of oxygen content, gaseous impurities in $\mathrm{Ni}$ likely constitute less than a few atomic percent. Such impurity estimates only include those incorporated into the lattice; impurities may also reside on grain boundaries or other defects and thus not affect XRD measurements of $\delta_{\mathrm{H}}$. 
In addition to stress changes contributed by $\Delta \sigma_{\mathrm{T}}(T)$ and $\sigma_{x}\left(\delta_{H}\right)$, several other mechanisms may be operative, particularly excess vacancy annihilation, void shrinkage, grain growth, and processes induced by solid-state amorphization. All but the last will make about the same contribution in both diffusion couple and single-layer elemental samples. The amorphization process may alter the stress in the Ni layer since vacancies are injected into that layer as the $\mathrm{Ni}$ atoms enter the amorphous phase. While there is an offset observed in the Ni phase between the curves of biaxial stress in single-layer elemental samples and diffusion couples, the evolution is parallel in all cases for both $\mathrm{Ni}$ and $\mathrm{Hf}$. For $\mathrm{Ni}$, despite the offset, which probably results from imperfect control of some deposition variable, the parallel evolution indicates that vacancy injection during SSAR does not contribute to the stress. One possible explanation of the absence of such a contribution is that yield or creep, possibly enhanced by the vacancy injection, limits the stress in the Ni layer at $T_{\text {ann }}$ (see below).

In $\mathrm{Ni}$, the stress at the annealing temperature, $\sigma\left(T_{\mathrm{ann}}\right)$, relaxes to small values [Fig. 2(a)]. The stress change observed at the longest anneal time is $+0.30 \pm 0.06 \mathrm{GPa}$, and the average change in $\sigma_{x}\left(\delta_{H}\right)$ from its initial value is $-0.15 \pm 0.09 \mathrm{GPa}$. Thus in $\mathrm{Ni}$, solute incorporation stresses are opposite in sign to the observed changes and other processes must dominate the stress relaxation at $T_{\mathrm{ann}}$. In Hf, observed stress changes also indicate stress relaxation at $T_{\text {ann }}$ [Fig. 2(b)]. The average change in $\sigma_{x}$ from its initial value is $-0.29 \pm 0.05 \mathrm{GPa}$, and the average change in $\sigma_{x}\left(\delta_{\mathrm{H}}\right)$ is $-0.17 \pm 0.03 \mathrm{GPa}$. Thus about $60 \%$ of the stress change in Hf is accounted for by the change in $\delta_{\mathrm{H}}$. Note that these changes are independent of SSAR. It is not surprising to see stress relief in Hf over long times at $T_{\mathrm{ann}}$ since, $T_{\mathrm{ann}} / T_{\mathrm{m}}=0.25\left(T_{\mathrm{m}}=\right.$ melting temperature), and the stress evolution data of Ref. 52 indicate significant relaxation occurs in $15 \mathrm{~min}$ at such homologous temperatures in Ni if $T_{\text {ann }}>T_{\text {dep }}=$ deposition temperature.

Except for the single-layer elemental $\mathrm{Ni}$ sample, which displays a constant offset, calculated $\sigma\left(T_{\text {ann }}\right)$ in the crystalline phases approach zero at the longest anneal times. Thus, stress relaxation in the terminal phases occurs at $T_{\text {ann }}$ on the time scale of tens of minutes in $\mathrm{Ni}$ and hundreds of minutes in Hf. Some of the mechanisms responsible for the observed stress changes are discussed below.

We now briefly discuss other mechanisms of postdeposition stress evolution in the crystalline layers at $T_{\mathrm{ann}}$ (see reviews by Hoffman ${ }^{53}$ and Doerner and $\mathrm{Nix}^{48}$ ). Possibly significant contributors include the annihilation of excess vacancies trapped during deposition, grain boundary void shrinkage, and grain growth (Ni only, based on our measured grain sizes, consistent with expectations ${ }^{53}$ ). No significant recrystallization is expected ${ }^{54}$ or ob- served. Vacancy annihilation may not significantly contribute to stress evolution in $\mathrm{Hf}$ at the annealing temperature. ${ }^{55}$

Hoffman has compiled ${ }^{53}$ stress values in as-deposited $1000-\AA$ Ni films, corrected to the deposition temperature, $T_{\text {dep }}$, ranging from +0.26 to $+0.8 \mathrm{GPa}$ (corrected for the difference between the measurement temperature and the deposition temperatures, $T_{\mathrm{obs}}$ and $T_{\mathrm{dep}}$, respectively). Even for similar substrates and temperatures, there is considerable scatter in the data. Stress relief is nearly complete after annealing for $15 \mathrm{~min}$ at $225^{\circ} \mathrm{C} .{ }^{52}$ These results are consistent with the initial stresses and the changes upon annealing we report for $\mathrm{Ni}$. There are fewer literature reports on stresses in $\mathrm{Hf}$, but the asdeposited stress is expected to be close to that of the chemically similar Zr. Under conditions similar to those of the present study, $\sigma=+0.7 \mathrm{GPa}$ was measured by a curvature-based method in as-deposited $\mathrm{Zr} .{ }^{56}$ Stress due to oxygen incorporation has been previously reported in several metals which, like Hf, have a high affinity for oxygen. ${ }^{57}$

To summarize the stress effects in crystalline layers: most-importantly, comparing single-layer elemental and diffusion couple samples shows that there are neither significant contributions due to dissolution of metal atoms nor of vacancy injection resulting from SSAR. In both $\mathrm{Ni}$ and $\mathrm{Hf}$ layers, stress changes consistent with relaxation at $T_{\text {ann }}$ are observed. In Ni layers, the contribution due to $\delta_{\mathrm{H}}$ is not dominant. While yield and/or creep are possible, excess vacancy annihilation, grain growth, and void shrinkage are likely processes enabling the stress relaxation in $\mathrm{Ni}$ layers. In Hf layers, roughly $60 \%$ of the observed stress changes are accounted for by the increasing $\delta_{\mathrm{H}}$, probably caused by gaseous impurity incorporation. Comparison between the observed and expected stress relaxation support our conclusion that uncertainties in stress evolution due to yield or relaxation during heatup or cooldown and at room temperature are small.

\section{Amorphous phase stress contribution}

Estimates of the contribution by the amorphous phase to the average film stress will now be considered. First, contributions of the crystalline phases, thermal expansion in the amorphous phase, and the volume change upon amorphization are evaluated. See above on the agreement between average stresses based on curvature and XRD measurements (Sec. IV. A).

In Fig. 3, the contribution of the stresses in the crystalline layers to the overall average stress is displayed. This contribution is first calculated using Eq. (2), the stress data of Fig. 2, and RBS thickness information (Fig. 4). Then, if no yield or stress relaxation takes place during cooldown, $\Delta \sigma_{\mathrm{T}}\left(T_{\mathrm{ann}}\right)$ gives the stress correction to 
$T_{\text {ann. }}$. The band of closely spaced parallel lines in Fig. 3 indicates the possible range if thermal stresses in Ni only or both $\mathrm{Ni}$ and $\mathrm{Hf}$ crystalline layers partially relax or yield during cooldown or at room temperature. (It is unlikely that stresses would relax or yield in the morerefractory Hf layers alone.) Recall that relaxation or yielding during heating are not expected. Note that the error due to incomplete knowledge of the behavior of the elemental phases during cooldown is likely to be similar for all data points. The contribution to variation in the corresponding curve in Fig. 3 due to the decreasing thickness of the elemental layers is smooth and relatively small. We conclude that the crystalline phases do not account for the large observed curvature changes.

The contribution of differential thermal expansion in the amorphous layer to the film average stress, measured at room temperature, has been estimated from mechanical characteristics of similar materials. ${ }^{11,58}$ The contribution of thermal stress in the amorphous layer to the overall average stress, used as a correction in Fig. 3, increases from its initial value of zero but remains small. While amorphous phase thermal stress values suffer from the same uncertainty in magnitude due to possible yield or relaxation as those of the crystalline phases, this uncertainty is very small and therefore does not introduce additional uncertainty in the stress evolution.

In epitaxial crystalline systems undergoing an interfacial reaction to form a crystalline compound, the sign of resulting stresses does not generally correlate with the volume change upon reaction; rather the sign is determined by lattice mismatch. ${ }^{59}$ However, this constraint may not apply to amorphous phase formation since a lattice no longer exists in the reaction products. Assuming that atomic mobility is sufficient to attain nearly the metastable equilibrium density, amorphization results in a volume change, $\Delta V_{\mathrm{a}}$. If the biaxial stress in the present case is due to this volume change under constraint by the substrate, a biaxial stress, $\sigma_{\Delta V}$, given by Eq. (3) with $\delta_{\mathrm{H}}=1 / 3 \Delta V_{\mathrm{a}} / V$, results. Using $\Delta V_{\mathrm{a}} / V=-3.1 \%$ for the similar Ni-Zr system ${ }^{14}$ and above values for amorphous phase properties gives $\sigma_{\Delta V_{\mathrm{a}}}=+2.6 \mathrm{GPa}$ in the amorphous phase.

If the stress in the growing amorphous layer relaxes by creep, its time-dependent contribution to the overall stress may be analyzed as follows. In a small volume element at time $t$ after its formation $\sigma=\sigma_{0} \exp (-\alpha t),{ }^{60}$ where $\sigma_{0}$ is the initial stress, $\alpha=E /\{6 \eta(1-v)\}$, and $\eta$ is the viscosity. The average stress in the amorphous layer which has grown to a thickness $x$ at time $t$ can be expressed by integrating over the contributions of all parts of the layer, as determined by their respective age $t-t^{\prime}$ :

$$
\bar{\sigma}(t)=\frac{\sigma_{0}}{x(t)} \int_{0}^{t} \exp \left[-\alpha\left(t-t^{\prime}\right)\right] \frac{\mathrm{d} x\left(t^{\prime}\right)}{\mathrm{d} t^{\prime}} \mathrm{d} t^{\prime}
$$

As apparent from the approximately linear plots of Fig. 4 and expected in diffusion-limited reactions, the amorphous layer thickness is proportional to $t^{1 / 2}$, and $t-t^{\prime}=$ $k\left(x^{2}-x^{\prime 2}\right)$. Under these conditions, the behavior of $x(t) \bar{\sigma}(t)$, the contribution of the amorphous phase to the observed force per unit length, is qualitatively similar to that observed in Fig. 3. Also, it can be shown numerically that $x(t) \bar{\sigma}(t)$ has a maximum at $t_{\max } \approx \alpha^{-1}$. Within the resolution of our data, $t_{\max } \approx 30 \mathrm{~min}$, yielding $\eta \approx$ $8 \times 10^{13} \mathrm{~Pa}$ s at $340{ }^{\circ} \mathrm{C}$, reasonably consistent with values of $\eta=(2-8) \times 10^{13} \mathrm{~Pa}$ s obtained by extrapolation to the same homologous temperature relative to the crystallization temperature for $\mathrm{Ni}_{x} \mathrm{Zr}_{100-x}, x=30$ to $50 .{ }^{61,62}$ The fact that the sample composition spans a wide range is not expected to qualitatively affect this comparison. From Figs. 3 and 4 and Eq. (4), we obtain $\sigma_{0} \approx 16 \mathrm{GPa}$. While this value is very large and not consistent with $\sigma_{\Delta V_{\mathrm{a}}}$, we note that it is on the order of the theoretical strength of $10 \mathrm{GPa}^{63}$ and that yield strengths are high in very thin layers because of the difficulty of nucleating shear bands. We do not currently have an explanation for the high estimate of $\sigma_{0}$.

\section{Irradiation effects}

In addition to being annealed in parallel, one of a pair of diffusion couples was irradiated with energetic ions to monitor the effects of altering the stress state on diffusion. As described above, care was taken to minimize other changes induced by irradiation and to maintain adequate controls. Despite the mild irradiation conditions, control samples are essential to distinguish irradiation from annealing effects. The primary control sample was an identical sample prepared and annealed together with the sample to be irradiated. The stress and reaction extent measurements (Figs. 3 and 4) show very good intersample reproducibility under these optimal conditions. By using a nominally bare substrate as a control (see Sec. II. A), we have determined that the observed curvature changes in the diffusion couple after both irradiation and subsequent annealing were dominated by stress changes in the metal layers.

We have measured immediate radiation effects on both stress and reaction extent. TRIM simulations ${ }^{24}$ indicate an insignificant degree of ballistic mixing for the ion species, energy, and dose used; the low temperature carefully maintained during irradiation is also expected to suppress radiation-enhanced diffusion. ${ }^{64}$ These expectations were confirmed by RBS depth-profiling both before and immediately after irradiation (Fig. 4, arrow). We also find that the irradiation did not affect the subsequent reaction rate, which is noteworthy in light of the evident impact on stress.

It is not a priori possible to state whether a given irradiation will increase or decrease the stress or free energy. Two competing effects are present: one is the 
introduction of defects and associated decrease in density, leading to a more compressive stress. The other is radiation-induced atomic mobility, expected to assist both stress relaxation and densification, thus lowering the total free energy. Given the low irradiation temperature and absence of radiation-enhanced diffusion, we suggest the following scenario. During irradiation, the film volume increased due to defect production, and a compressive stress developed which was relieved by yield or creep. Upon heatup, defect annihilation led to densification and therefore a stress more tensile than before irradiation. This suggested mechanism is applicable to both crystalline and amorphous phases; in the latter, defects are excess free volume. Any radiation-induced defects seem to be quickly eliminated upon annealing and do not impact the reaction rate; see Fig. 4. Note that the compressive stress due to volume increase must overcome the 0.36 and $0.12 \mathrm{GPa}$ thermal stresses, respectively, in $\mathrm{Ni}$ and amorphous alloy layers resulting from cooling to $80 \mathrm{~K}$ before the process we suggest can result in a more tensile stress upon postirradiation return to room temperature. On the other hand, in the Hf layer, this process would be slightly enhanced by the $-0.06 \mathrm{GPa}$ thermal stress at $80 \mathrm{~K}$. Our observations suggest that either irradiationinduced stress changes in the Ni layer are negligible or that such changes relax upon subsequent annealing.

In summary, relaxation of thermal stresses alone cannot account for the stress changes in the metal film due to irradiation. We suggest that these changes are due to relaxation of thermal and defect-related stresses during irradiation, followed by defect annihilation upon heating. The Ni layer does not contribute to these changes, and the Hf layer accounts for about $40 \%, 0.18 \mathrm{GPa}$. If $\tilde{D}$ is sensitive to the free volume in the amorphous alloy, any possible change in the free volume is annihilated in the initial stages of annealing and has little impact on $\tilde{D}$.

The most important difference between the present work and that of Aaen Andersen et al. ${ }^{6-8}$ is that slowing of the reaction at long times is not significant in the present work. It is difficult to compare our results with theirs since they do not report the irradiation temperature. Further, the large radiation damage level we calculate for their dose, $17 \mathrm{dpa}$, raises the possibility of other microstructural changes besides structural relaxation. While the interpretation presented in Refs. 6-8 explaining a postirradiation enhancement in the SSAR rate remains unproven, it is not inconsistent with the present work. Further work to demonstrate the effect of irradiation on thermodynamics and kinetics in the amorphous phase will be required.

\section{E. Diffusion kinetics}

Since the early observations of SSAR in Ni-Hf by Van Rossum et al., ${ }^{4}$ a diffusion-controlled reaction mechanism at short times has been inferred from the observa- tion of a $t^{1 / 2}$ growth law. ${ }^{3}$ In Ref. 4 , the interdiffusion coefficient in a diffusion couple was reported as $\tilde{D}=$ $6 \times 10^{-19} \mathrm{~m}^{2} / \mathrm{s}$ at $340{ }^{\circ} \mathrm{C}$. Recalculation for these data, on the basis of common-tangent compositions we have determined, ${ }^{2}$ yields $\tilde{D}=2 \times 10^{-19} \mathrm{~m}^{2} / \mathrm{s}$, in good agreement with $1.6 \times 10^{-19} \mathrm{~m}^{2} / \mathrm{s}$ determined from Fig. 4 . Discrepancies among reported common-tangent compositions have been discussed elsewhere. ${ }^{2}$

For long SSAR reaction times in Ni-Hf or the similar $\mathrm{Ni}-\mathrm{Zr}$ system, Van Rossum ${ }^{4}$ and others ${ }^{5-9}$ have reported a slowing in the reaction-rate law. In the work with reaction conditions most-comparable to the present, ${ }^{4}$ some slowdown already becomes apparent after $0.5 \mathrm{~h}$ of annealing and is pronounced after $1 \mathrm{~h}$. While several proposals have been made in attempting to explain the rate law changes, no one proposal has yet proved entirely satisfactory. It has been reported that $\mathrm{Hf}+\mathrm{Ni}$ diffusion couples ( $\mathrm{Ni}$ as the surface layer) react more slowly or require higher temperatures to begin SSAR than $\mathrm{Ni}+\mathrm{Hf}$ diffusion couples (Hf as surface layer). ${ }^{4}$ The explanation given was contamination of the diffusion region by oxygen which diffused through $\mathrm{Ni}$ when that element was on the surface. In contrast, a native oxide on the surface of $\mathrm{Ni}+\mathrm{Hf}$ diffusion couples was suggested to act as a barrier for further in-diffusion of oxygen. In the present work, great care was taken to avoid significant oxidation, and the reaction rate decrease at longer times was found to be less than that previously observed. ${ }^{4}$ The initially greater reaction rate in the $\mathrm{Hf}+\mathrm{Ni}$ versus the $\mathrm{Ni}+\mathrm{Hf}$ diffusion couple is not likely significant since the samples evolve in parallel after the first $0.5 \mathrm{~h}$. Thus, while oxygen contamination may be a cause of previously-observed reaction slowing, it is likely to be significantly lower in the present work.

That there may be some difference in adherence at the substrate-Ni interface compared to the substrate-Hf interface, and thus different degrees of relaxation, was considered. However, significant stress differences were not observed with differing layer orders. Therefore, stresses in the amorphous layers are unlikely to depend on the layer order, and the early-stage reaction-rate differences are probably due to minor variations in deposition conditions.

In a recent study on crystalline $\mathrm{Ni}+$ amorphous $\mathrm{Ni}_{50} \mathrm{Zr}_{50}$ multilayers, Karpe et al. ${ }^{65}$ report $\theta / 2 \theta$ XRD measurements of SSAR rate and strain in the Ni layers. Values of $\tilde{D}$ are reported to decrease both at longer anneal times and for shorter multilayer periods. We emphasize here that the evolution of stress in one layer does not directly provide information on a neighboring layer. The stresses in individual layers can evolve essentially independently, since the thick substrate balances the net force per unit length in the entire film. ${ }^{48}$ It is difficult to determine stresses from the $\mathrm{Ni}$ strains reported in Ref. 65, 
since they are based on only a single Bragg peak in a single orientation; further measurements will be necessary to determine the stress in the amorphous layers.

We now address the cause for the absence of a stress effect on interdiffusion. A change in free energy of one of the phases due to stress would shift the commontangent composition, providing a different boundary condition for diffusion and affecting the diffusional flux. For example, if the free energy of the amorphous phase is raised, then its homogeneity range when in metastable equilibrium with the terminal phases shrinks. For a given thickness of the amorphous layer, the concentration gradient will decrease, thus lowering the diffusional flux. In the present system, since the driving force is large, as required for SSAR, the contribution of the stress term to the driving force is negligible. In light of the experimental results, we conclude that the stress effect on atomic mobility is also negligible.

\section{CONCLUSIONS}

During amorphization in a Ni-Hf diffusion couple, a large tensile stress develops, which goes through a maximum and then decreases to an approximately steady-state value. Irradiation of a reacting diffusion couple induces additional tensile stress which increases further and then decays upon subsequent annealing. The contributions of the crystalline phases, differential thermal expansion of the amorphous phase, and interfaces to the observed stress changes are all small, and the growth stress in the amorphous phase dominates. The tensile stress in the amorphous phase is suggested to be due to the volume change upon reaction and to relax by creep. Neither the large stress changes accompanying SSAR nor those induced by ion irradiation are correlated with noticeable changes in the reaction kinetics. Oxygen contamination is less significant than in previously published studies, in which it may have been the cause of reaction slowing. While some slowing from diffusion-limited kinetics is observed, it is not explained by macroscopic stress changes. As background, further work is necessary on factors influencing possible disagreement between XRD and curvature measurements of stress. ${ }^{47}$ Meanwhile, great caution should be exercised in basing conclusions on the differences between stress values obtained by the two techniques.

\section{ACKNOWLEDGMENTS}

This work was funded by the United States National Science Foundation Grant Nos. DMR-92000132 and DMR-9500617. One of the authors (M.A.) gratefully acknowledges a fellowship of the Engineering and Physical Sciences Research council of the United Kingdom while on leave at the University of Cambridge. Thanks to Teledyne Wah Chang Corp. for graciously supplying low-Zr high-purity hafnium and to Dr. C. Kalnas and Dr. L. Parfitt Kendig for providing cover slip substrates selected for flatness. Samples were prepared, irradiated, and analyzed by RBS at the Michigan Ion Beam Laboratory at the University of Michigan.

\section{REFERENCES}

1. W.L. Johnson, Prog. Mater. Sci. 30, 81 (1986). Compare for example, W.L. Johnson, B. Dolgin, and M. Van Rossum, in GlassCurrent Issues, edited by A.F. Wright and J. Dupay (NATO ASI Series, E-92, Martinus Nijhoff, Boston, MA, 1985), p. 172.

2. W.S.L. Boyer and M. Atzmon, J. Alloys Compd. 194, 213 (1993).

3. G.V. Kidson, J. Nucl. Mater. 3, 21 (1961), and references therein.

4. M. Van Rossum, M-A. Nicolet, and W.L. Johnson, Phys. Rev. B 29, 5498 (1984).

5. K.M. Unruh, W.J. Meng, and W.L. Johnson, in Layered Structures, Epitaxy, and Interfaces, edited by J.M. Gibson and L.R. Dawson (Mater. Res. Symp. Proc. 37, Pittsburgh, PA, 1985), p. 551.

6. L-U. Aaen Andersen, J. Bøttiger, J. Janting, N. Karpe, K.K. Larsen, A.L. Greer, and R.E. Somekh, Mater. Sci. Eng. A 133, 415 (1991)

7. N. Karpe, J. Bøttiger, A.L. Greer, J. Janting, and K. Kyllesbech Larsen, J. Mater. Res. 7, 926 (1992).

8. A.L. Greer, Mater. Sci. Eng. A 134, 1268 (1991).

9. G.B. Stephenson, J. Non-Cryst. Solids 66, 393 (1984).

10. S.D. Theiss, F. Spaepen, and M.J. Aziz, in Thin Films: Stresses and Mechanical Properties V, edited by S.P. Baker, P. Børgensen, P.H. Townsend, C.A. Ross, and C.A. Volkert (Mater. Res. Soc. Symp. Proc. 356, Pittsburgh, PA, 1995), p. 15.

11. M. Moske and K. Samwer, Z. Phys. B 77, 3 (1989).

12. M. Moske and K. Samwer, in Thin Films: Stresses and Mechanical Properties $V$, edited by S.P. Baker, P. Børgensen, P.H. Townsend, C.A. Ross, and C.A. Volkert (Mater. Res. Soc. Symp. Proc. 356, Pittsburgh, PA, 1995), p. 27.

13. M.J. Aziz, P.C. Sabin, and G-Q. Lu, Phys. Rev. B 44, 9812 (1991); compare references therein.

14. Z. Altounian and J.O. Strom-Olsen, Phys. Rev. B 27, 4149 (1983).

15. P. Van Houtte and L. De Buyser, Acta Metall. Mater. 41, 323 (1993).

16. A.L. Greer, N. Karpe, and J. Bøttiger, J. Alloys Compd. 194, 199 (1993).

17. F.L. Yang, W.C. Shih, and A.L. Greer, in Thin Films: Stresses and Mechanical Properties V, edited by S.P. Baker, P. Børgensen, P.H. Townsend, C.A. Ross, and C.A. Volkert (Mater. Res. Soc. Symp. Proc. 356, Pittsburgh, PA, 1995), p. 21.

18. G.B. Stephenson, Acta Metall. 36, 2663 (1988)

19. I.C. Noyan and C.C. Goldsmith, Adv. X-ray Anal. 33, 137 (1990).

20. S.G. Malhotra, Z.U. Rek, S.M. Yalisov, and J.C. Bilello, Thin Solid Films 301, 45 (1997).

21. S.G. Malhotra, Z.U. Rek, S.M. Yalisov, and J.C. Bilello, Thin Solid Films 301, 55 (1997).

22. I.C. Noyan, T.C. Huang, and B.R. York, Crit. Rev. Solid State Mater. Sci. 20, 125 (1995).

23. A. Kinbara and H. Haraki, J. Appl. Phys. Japan 2, 328 (1965).

24. J.F. Ziegler, J.P. Biersack, and U. Littmark, The Stopping and Range of Ions in Solids (Pergamon Press, New York, 1985), Vol. 1.

25. V. Chinellato, V. Gottardi, S. Lo Russo, P. Mazzoldi, F. Nicoletti, and P. Polato, Radiat. Effects 65, 31 (1982).

26. G.W. Arnold, Radiat. Effects 98, 55 (1986).

27. L.R. Doolittle, Nucl. Instrum. Methods B 9, 334 (1985).

28. R. Feder and B.S. Berry, J. Appl. Crystallogr. 3, 372 (1970). 
29. M. Mack and W. Parrish, Acta Crystallogr. 23, 693 (1967).

30. M.M. Hall, V.G. Veeraraghavan, H. Rubin, and P.G. Winchell, J. Appl Crystallogr. 10, 66 (1977).

31. J.F. Nye, Physical Properties of Crystals, their Representation by Tensors and Matrices (Clarendon Press, Oxford, United Kingdom, 1964).

32. W. Boyer and M. Atzmon (unpublished).

33. B.M. Clemens and J.A. Bain, MRS Bull. 17, 46 (1992).

34. G. Cornella, S-H. Lee, W.D. Nix, and J.C. Bravman, Appl. Phys. Lett. 71, 2949 (1997).

35. H.P. Klug, L.E. Alexander, X-ray Diffraction Procedures For Polycrystalline and Amorphous Materials, 2nd ed. (John Wiley \& Sons, New York, 1974).

36. J.D. Finegan and R.W. Hoffman, in Eighth National Symposium on Vacuum Technology Transactions (Pergamon Press, New York, 1962), p. 935.

37. P.A. Flinn, D.S. Gardner, and W.D. Nix, IEEE Trans. Electron Devices, ED-34, 689 (1987).

38. F.J. von Preissig, J. Appl. Phys. 66, 4262 (1989).

39. W. Jost, Diffusion in Solids, Liquids, Gases (Academic Press, New York, 1960). Equation [1.334] has a typographical error; it should read $D_{I I}=\left(\xi^{\prime}\right)^{2} / 4 \gamma^{\prime 2} t$; likewise in [1.335], $\gamma^{\prime \prime}$ should be $\gamma^{\prime \prime 2}$.

40. J.A. Ruud, A. Witvrouw, and F. Spaepen, J. Appl. Phys. 74, 2517 (1993).

41. J.A. Bain, L.J. Chyung, S. Brennan, and B.M. Clemens, Phys. Rev. B 44, 1184 (1991).

42. M.A. Hollanders, B.J. Thijsse, and E.J. Mittemeijer, Phys. Rev. B 42, 5481 (1990).

43. I.C. Noyan and J.B. Cohen, Residual Stress: Measurement by Diffraction and Interpretation (Springer-Verlag, New York, 1987).

44. R. Venkatraman, P.R. Besser, J.C. Bravman, and S. Brennan, J. Mater. Res. 9, 328 (1994).

45. C.V. Thompson, in Polycrystalline Thin Films-Structure, Texture, Properties and Applications, edited by K. Barmak, M.A. Parker, J.A. Flow, R. Sinclair, and D.A. Smith (Mater. Res. Soc. Symp. Proc. 343, Pittsburgh, PA, 1994), p. 3.

46. B.D. Cullity, Elements of X-ray Diffraction (Addison-Wesley, Reading, MA, 1956).
47. D.B. Knorr, J. Met. 44, 29 (1992).

48. M.F. Doerner and W.D. Nix, CRC Crit. Rev. Solid State Mater. Sci. 14, 225 (1988). Additional reviews are cited therein.

49. Smithells Metals Reference Book, edited by E.A. Brandes and G.B. Brook (Butterworth-Heinemann Ltd., London, United Kingdom, 1992).

50. Fisher Scientific, Pittsburgh, PA (personal communication).

51. P. Villars and L.D. Calvert, Pearson's Handbook of Crystallographic Data for Intermetallic Phases, 2nd ed. (ASM International, Materials Park, OH, 1991).

52. R.W. Hoffman, R.D. Daniels, and E.C. Crittenden, Jr., Proc. Phys. Soc. 64, 497 (1954).

53. R.W. Hoffman, Phys. Thin Films 3, 211 (1966).

54. R. Schulz, M.L. Trudeau, and A. van Neste, Mater. Sci. Eng. A A134, 1354 (1991).

55. Y.T. Cheng, M.A. Nicolet, and W.L. Johnson, in Thin-FilmsInterfaces and Phenomena, edited by R.J. Nemanich, P.S. Ho, and S.S. Lau (Mater. Res. Soc. Symp. Proc. 54, Pittsburgh, PA, 1986), p. 174.

56. E. Klokholm and B.S. Berry, J. Electrochem. Soc. 115, 823 (1968).

57. F.M. d'Heurle, Int. Mater. Rev. 34, 53 (1989).59

58. H.U. Künzi, Glassy Metals II, edited by H. Beck and H-J. Güntherodt (Springer, New York, 1983), p. 174.

59. P.P. Bauad, F.M. d'Heurle, S. Chevacharoenkul, and E.A. Irene, J. Vac. Sci. Technol. B 11, 304 (1993); F.M. d'Heurle, Defect Diffus. Forum 129-130, 137 (1996).

60. A. Witvrouw, C.A. Volkert, and F. Spaepen, Mater. Sci. Eng. A 134, 1274 (1991).

61. K. Russew, F. Sommer, P. Duhaj, and I. Bakonyi, J. Mater. Sci. 27, 3565 (1992).

62. F. Sommer, T. Lang, and B. Predel, Z. Metall. 78, 648 (1987).

63. F. Spaepan, Rapidly Quenched Metals III, edited by B. Cantor (Metals Society, London, United Kingdom, 1978), p. 253.

64. F. Ding, R.S. Averback, and H. Hahn, J. Appl. Phys. 64, 1785 (1988).

65. N. Karpe, J. Bøttiger, J.P. Krog, J.S. Conyers, A.L. Greer, and R.E. Somekh, Philos. Mag. A 75, 461 (1997). 